\title{
Long Range Atomic Potentials in Thomas-Fermi Theory
}

\author{
Haim Brezis ${ }^{1}$ and Elliott H. Lieb ${ }^{2 \star}$ \\ ${ }^{1}$ Département de Mathématiques, Université Paris VI, F-75230 Paris Cedex 05, France \\ 2 Departments of Mathematics and Physics, Princeton University, Princeton, New Jersey 08540, USA
}

\begin{abstract}
We prove that the interaction among neutral atoms in ThomasFermi theory behaves, for large separation $l$, like $\Gamma l^{-7}$. The constant $\Gamma$ is independent of the atomic nuclear charges, but does depend on the relative positions of the nuclei. We also show that $\Gamma$ is not a simple sum of pair terms, i.e. in TF theory three and higher body terms persist into the asymptotic (in l) region.
\end{abstract}

\section{Introduction}

We investigate the interaction among neutral atoms in Thomas-Fermi (TF) theory and prove that the interaction is asymptotically (const.) $l^{-7}$ for large separation, $l$. The non-neutral case will not be discussed because there the interaction is asymptotically (const.) $l^{-1}$ for elementary electrostatic reasons.

The TF theory is defined as follows (see' [1] for a complete discussion and the basic theorems): Define the functional

$$
\begin{aligned}
\mathscr{E}(\varrho) & =\frac{3}{5} \int \varrho(x)^{5 / 3} d x-\int V(x) \varrho(x) d x+\frac{1}{2} \iint \varrho(x) \varrho(y)|x-y|^{-1} d x d y+U \\
V(x) & =\sum_{j=1}^{k} z_{j}\left|x-R_{j}\right|^{-1} \\
U & =\sum_{1 \leqq i<j \leqq k} z_{i} z_{j}\left|R_{i}-R_{j}\right|^{-1}
\end{aligned}
$$

for positive functions $\varrho(x)$ on $\mathbb{R}^{3}$ in $L^{5 / 3} \cap L^{1}$. $\mathscr{E}(\varrho)$ is the functional appropriate to a system consisting of $k$ nuclei of charges $z_{i}>0$ and located at $R_{i} \in \mathbb{R}^{3}$. The TF energy is defined to be

$$
e\left(z_{1}, \ldots, z_{k} ; R_{1}, \ldots, R_{k}\right)=\min _{\varrho} \mathscr{E}(\varrho)
$$

* Work partially supported by U.S. National Science Foundation grant MCS 7521684 A02 
which we shall frequently denote simply by $e(\mathbf{z} ; \mathbf{R})$. The important fact is that there is a unique function $\varrho$ which minimizes $\mathscr{E}(\varrho)$. It has the property that

$$
\int \varrho(x) d x=Z \equiv \sum_{j=1}^{k} z_{j}
$$

(i.e. neutrality) and satisfies the TF equation

$$
\varrho^{2 / 3}(x)=\phi(x) \equiv V(x)-\int \varrho(y)|x-y|^{-1} d y .
$$

This $\varrho$ is also the unique non-negative solution to (1.4). Equivalently

$$
-(4 \pi)^{-1} \Delta \phi+\phi^{3 / 2}=\sum_{j=1}^{k} z_{j} \delta\left(x-R_{j}\right) .
$$

We want to investigate $e(\mathbf{z} ; \mathbf{R})$ as the $\left|R_{i}\right| \rightarrow \infty$. It is relatively easy to prove that $e(\mathbf{z} ; \mathbf{R}) \rightarrow \sum_{j=1}^{k} e^{\mathrm{at}}\left(z_{j}\right)$ as $\left|R_{i}\right| \rightarrow \infty$ and $\left|R_{i}-R_{j}\right| \rightarrow \infty$, all $i \neq j$, where $e^{\mathrm{at}}(z)$ is the energy (1.2) for an atom, i.e. $k=1, z_{1}=z$ (which is obviously independent of $R_{i}$ ). Hence we consider

$$
f(l) \equiv e(\mathbf{z} ; l \mathbf{R})-\sum_{i=1}^{k} e^{\mathrm{at}}\left(z_{i}\right)
$$

where $l \mathbf{R}=\left(l R_{1}, \ldots, l R_{k}\right)$, corresponding to a uniform dilation of the molecule.

We will prove two principal results: If we write

$$
f(l)=C(l, \mathbf{z}, \mathbf{R}) l^{-7}
$$

then

$$
\begin{aligned}
& \text { (A) } C(l, \mathbf{z}, \mathbf{R}) \quad \text { is an increasing function of } l, \\
& \text { (B) } \lim _{l \rightarrow \infty} C(l, \mathbf{z}, \mathbf{R}) \equiv \Gamma(\mathbf{R})>0, \quad \Gamma(\mathbf{R}) \quad \text { finite }
\end{aligned}
$$

exists. $\Gamma$ is independent of the nuclear charges $\mathbf{z}$ !

It is amusing to note that in the quantum theory there is also an $l^{-7}$ long range (van der Waals) interaction for closed shell atoms provided retardation effects in the Coulomb interaction are taken into account. However, the sign of $\Gamma(\mathbf{R})$ is negative instead of positive in the quantum theory and its physical basis is unrelated to that of the TF theory [9].

The fact that $\Gamma(\mathbf{R})$ is independent of $\mathbf{z}$ in TF theory comes essentially from the following fact: The $Q^{\text {at }}(x)$ for a neutral atom located at $R=0$ is asymptotically given by Sommerfeld's formula [5] (see also [1]) $\varrho(x) \approx(3 / \pi)^{3}|x|^{-6}$, which is independent of $z$. The $l^{-7} \Gamma$ interaction comes, in some sense, from the overlap of the tails of the atomics $\varrho$ 's.

Previously obtained, related theorems include the following: (a) Teller's theorem [6] (see [1]) which says that $f(l)>0$ for all $l$; (b) The theorem [2] that if $e(\mathbf{z} ; \mathbf{R})$ is decomposed (by means of successive substractions) into 2,3 , 4, etc. body terms then the even-body terms are all positive and the odd-body terms are all negative (assuming all $z_{i}>0$ ); (c) The theorem [3] that the pressure and 
compressibility are positive, i.e. $f(l)$ is a decreasing, convex function of $l$ (the convexity theorem is stronger than the statement that the compressibility is positive).

We are aware of only two previous analyses about the asymptotic nature of $f(l)$. One is a preprint [4] by Lee, Longmire and Rosenbluth in which a numerica] evaluation of $f(l)$ is carried out for two atoms of equal nuclear charge. They were unable to determine the asymptotic form of $f(l)$, but estimated that $f(l) \approx \mathrm{cl}^{-7}$. The other is a variational calculation by Roberts [10] of the same homonuclear system. He derived variational upper and lower bounds to $f(l)$ using (1.1) and Firsov's principle [11]. The two bounds had a common term $f_{0}(l)$ and different additional terms $\approx l^{-7}$. Roberts claimed that $f_{0}(l) \approx l^{-6}$. If so, this would violate the theorem of our paper. Upon closer examination, however, we were able to show that $f_{0}(l) \approx l^{-7}$, thus reestablishing consistency with our result.

We will also show that $\Gamma(\mathbf{R})$, for $k \geqq 3$, is not a sum of pair terms, i.e.

$$
\Gamma\left(R_{1}, \ldots, R_{k}\right) \neq \sum_{1 \leqq i<j \leqq k} \Gamma_{2}\left(R_{i}, R_{j}\right),
$$

where $\Gamma_{2}$ is the $\Gamma$ for two atoms. All the many body terms are present, i.e. they all have the same (non-zero) $l^{-7}$ dependence. By (b) above (Ref. [2]) we know the sign of these higher-body terms. In the statistical mechanics of many-body systems it is customary to assume that only two-body interactions are present, especially for large distances. In TF theory this is not so. Admittedly, TF theory is not the same as the correct quantum theory based on the Schrödinger equation, but it is not entirely unrelated to it. In particular the TF energy is asymptotically equal to the correct quantum energy as the $z_{i} \rightarrow \infty$, all $i$ (see [1]). Therefore the result obtained here may not be entirely without significance.

First we will prove that $l^{7} f(l)$ is bounded above (we know it is $>0$ by Teller's lemma). This upper bound is obtained by a variational calculation in Sect. II. It is simpler than Roberts' [10].

In Sect. III, Theorem (A) (1.8) will be proved. The results of Sect. II and III imply Theorem (B) (1.9). Section III also contains two different formulas for $\Gamma(\mathbf{R})$. These are not very explicit, however, for they require the solution of some TF equations; for the second formula this TF equation has strong singularities. Some mathematical aspects of these equations are given in the appendix.

Section IV contains the proof that $\Gamma(\mathbf{R})$, for $k \geqq 3$, is not a sum of pair terms.

\section{Upper Bound for Molecular Energy Differences}

Here we obtain an upper bound for $f=e(\mathbf{z} ; \mathbf{R})-\sum_{j=1}^{k} e^{\mathrm{at}}\left(z_{j}\right)$ by a variational calculation. Let $B_{1}, \ldots, B_{k}$ be balls centered respectively at $R_{1}, \ldots, R_{k}$, of radii $A_{1}, \ldots, A_{k}$ and such that the $B_{i}$ are disjoint. Let $\varrho_{i}(x)$ be the atomic, neutral TF density for an atom of charge $z_{i}$ located at $R_{i}$. If $\chi_{i}$ is the characteristic function of $B_{i}$, set $\varrho_{i}(x)=\varrho_{i}(x) \chi_{i}(x)$ and $\tilde{\varrho}_{i}(x)=\varrho_{i}(x)\left[1-\chi_{i}(x)\right]$. As variational $\varrho$ for the molecule take $\hat{\varrho}(x)=\sum_{j=1}^{k} \hat{\varrho}_{j}(x)$. Although $\int \hat{\varrho}<Z$, it is still true that $\mathscr{E}(\hat{\varrho}) \geqq e(\mathbf{z} ; \mathbf{R})$ because 
$e(\mathbf{z} ; \mathbf{R})$ is the absolute minimum of $\mathscr{E}(\varrho)$. Thus $f \leqq \mathscr{E}(\varrho)-\sum_{j=1}^{k} e^{\mathrm{at}}\left(z_{j}\right) \equiv g$. Since $\hat{\varrho}_{i}(x) \hat{\varrho}_{j}(x)=0$ for $i \neq j$, an easy computation (bearing in mind the TF equation for neutral atoms and Newton's theorem for non-overlapping spherical charge distributions) gives

$$
f \leqq g=\sum_{j=1}^{k} \Delta_{j}+\sum_{1 \leqq i<j \leqq k} Q_{i} Q_{j}\left|R_{i}-R_{j}\right|^{-1},
$$

where

$$
\begin{aligned}
& \Delta_{j}=\frac{2}{5} \int \tilde{\varrho}_{j}(x)^{5 / 3} d x+\frac{1}{2} \iint \varrho_{\varrho_{j}}(x) \varrho_{j}(y)|x-y|^{-1} d x d y \\
& Q_{j}=\int \tilde{\varrho}_{j}(x) d x .
\end{aligned}
$$

Since $\varrho_{j}(x)<(3 / \pi)^{3}\left|x-R_{j}\right|^{-6}$ for all $x$ (see [1]), this latter function can be used in (2.2) and (2.1). Thus

$$
\begin{aligned}
& Q_{j}<(6 / \pi)^{2} A_{j}^{-3}, \\
& \Delta_{j}<3^{5} \pi^{-4}(68 / 35) A_{j}^{-7} .
\end{aligned}
$$

If (2.3) is inserted in (2.1) and if the $R_{j}$ are replaced by $l R_{j}$ (and likewise the $A_{j}$ by $\left(A_{j}\right)$, we see that

$$
f \leqq C\left(R_{1}, \ldots, R_{k}\right) l^{-7} .
$$

Note that (2.4), the bound (2.1), with the values (2.3) is independent of the $z_{j}$ for all $R_{1}, \ldots, R_{k}$, not just large $R_{1}, \ldots, R_{k}$.

For two atoms separated by a distance $R$ we can choose $A_{1}=A_{2}=R / 2$ and obtain [using (2.1) and (2.3)]

$$
f=e^{\text {molecule }}-\sum_{j=1}^{2} e^{\text {at }}\left(z_{j}\right) \leqq R^{-7}(3 / \pi)^{4} 2^{11}(43 / 35) .
$$

\section{The Interaction at Large Distances}

As before $f(l) \equiv e(\mathbf{z} ; l \mathbf{R})-\sum_{j=1}^{k} e^{\text {at }}\left(z_{j}\right)$.

By scaling [1] one has that

$$
e(\mathbf{z} ; \mathbf{R})=l^{-7} e\left(l^{3} \mathbf{z} ; \mathbf{R}\right) \text {. }
$$

Thus

$$
C(l, \mathbf{z}, \mathbf{R}) \equiv l^{7} f(l)=e\left(l^{3} \mathbf{z} ; \mathbf{R}\right)-\sum_{j=1}^{k} e^{\mathrm{at}}\left(l^{3} z_{j}\right) .
$$

Our problem is to show that the right side of (3.2) has a limit as $l \rightarrow \infty$. In fact we will show that

$$
h(\mathbf{z}) \equiv e(\mathbf{z} ; \mathbf{R})-\sum_{j=1}^{k} e^{\mathrm{at}}\left(z_{j}\right)
$$


is an increasing function of $\mathbf{z}$ [in the sense that $h(\mathbf{z}) \geqq h\left(\mathbf{z}^{\prime}\right)$ if $\left.z_{i} \geqq z_{i}^{\prime}, i=1, \ldots, k\right]$. Thus, by Sect. II, $h(\mathbf{z})$ has a well defined, finite limit as $\min \left\{z_{j}\right\} \rightarrow \infty$. We call this limit $\Gamma(\mathbf{R})$. Since $h$ is increasing, $C(l, \mathbf{z}, \mathbf{R})$ is increasing in $l$. Hence $\Gamma(R)>0$ because $C$ is not identically zero.

We use the fact that $e(\mathbf{z} ; \mathbf{R})$ is differentiable in $\mathbf{z}[1]$ (in fact it is at least $C^{2}$ in $\mathbf{z}$ [3]) and

$$
e_{i} \equiv \partial e / \partial z_{i}=\lim _{x \rightarrow R_{i}}\left[\phi(\mathbf{z} ; \mathbf{R} ; x)-z_{i}\left|x-R_{i}\right|^{-1}\right],
$$

where $\phi(\mathbf{z} ; \mathbf{R} ; x)$ is the TF potential for the neutral molecule at the point $x \in \mathbb{R}^{3}$. Therefore

$$
h_{i}=\partial h / \partial z_{i}=\lim _{x \rightarrow R_{i}}\left[\phi(\mathbf{z} ; \mathbf{R} ; x)-\phi^{\mathrm{at}}\left(z_{i} ; R_{i} ; x\right)\right] .
$$

This is positive (unless $z_{j}=0$ for all $j \neq i$ ) by the strong form of Teller's lemma ([1], Theorem V.6).

We can now derive explicit formulae for $\Gamma(\mathbf{R})$. The first is to consider $h(\lambda \mathbf{z})$. Differentiating with respect to $\lambda$, using (3.5), and then integrating gives

$$
\Gamma(\mathbf{R})=\int_{0}^{\infty} d \lambda\left\{\sum_{j=1}^{k} z_{j} \lim _{x \rightarrow R_{j}}\left[\phi(\lambda \mathbf{z} ; \mathbf{R} ; x)-\phi^{\mathrm{at}}\left(\lambda z_{j} ; R_{j} ; x\right)\right]\right\} .
$$

It is not obvious from this formula that $\Gamma(\mathbf{R})$ is independent of $\mathbf{z}$. But we know that it is independent of $\mathbf{z}$ (provided all $z_{i}>0$ ) by the previous remark (monotonicity of $\mathbf{z} \rightarrow h(\mathbf{z})$ ). Therefore we can take $\mathbf{z}=\mathbf{z}^{0}=(1, \ldots, 1)$ on the right side of (3.6). Having done so, it is then obvious from (3.6) that $\Gamma(\mathbf{R})$ is a symmetric function of $R_{i}$.

A second advantage of formula (3.6) is that we will use it in Sect. IV to show that $\Gamma(\mathbf{R})$ is not simply a sum of pair terms (for $k \geqq 3$ ). From [2] we know that the 3,5 , etc. body terms are $\leqq 0$ and the 2,4 , etc. body terms are $\geqq 0$. The point we want to emphasize is that all these terms are non-zero for large $l$ in the sense that they all have the same $l^{-7}$ behavior.

Next we present another formula for $\Gamma(\mathbf{R})$ which is quite unsymmetric in the $R_{i}$ but which leads to an amusing variation of the TF equation in which strong singularities appear. First suppose $k=2$ and consider

$$
\begin{aligned}
h_{1} & =\partial h / \partial z_{1}=\lim _{x \rightarrow R_{1}}\left\{\phi\left(z_{1}, z_{2} ; R_{1}, R_{2} ; x\right)-\phi^{\mathrm{at}}\left(z_{1} ; R_{1} ; x\right)\right\} \\
& \equiv \psi_{1}\left(z_{1}, z_{2} ; R_{1}, R_{2}\right) .
\end{aligned}
$$

Then

$$
h(\mathbf{z})=\int_{0}^{z_{1}} d s_{1} \psi_{1}\left(s_{1}, z_{2} ; R_{1}, R_{2}\right),
$$

because $h=0$ when $z_{1}=0$. Now, for $x \neq R_{1}, R_{2}, \phi\left(z_{1}, z_{2} ; R_{1}, R_{2} ; x\right)$ is increasing in $z_{2}$ (by Teller's lemma). Let

$$
\phi\left(z_{1}, \infty ; R_{1}, R_{2} ; x\right)=\lim _{z_{2} \rightarrow \infty} \phi\left(z_{1}, z_{2} ; R_{1}, R_{2} ; x\right) .
$$


We will prove in the appendix that the limit in (3.8) is finite, and it will be identified as the solution of a TF equation with prescribed singularities at $R_{1}$ and $R_{2}$. Moreover (Prop. A 5), as $z_{2} \uparrow \infty$,

$$
\begin{aligned}
& \psi_{1}\left(z_{1}, z_{2} ; R_{1}, R_{2}\right) \uparrow \psi_{1}\left(z_{1}, \infty ; R_{1}, R_{2}\right) \\
& \quad \equiv \lim _{x \rightarrow R_{1}}\left[\phi\left(z_{1}, \infty ; R_{1}, R_{2} ; x\right)-\phi^{\mathrm{at}}\left(z_{1} ; R_{1} ; x\right)\right] .
\end{aligned}
$$

Given this fact, and the monotone convergence theorem,

$$
\Gamma(\mathbf{R})=\int_{0}^{\infty} d s_{1} \psi_{1}\left(s_{1}, \infty ; R_{1}, R_{2}\right) .
$$

The case $k>2$ can be handled in a similar way. We illustrate this for $k=3$. Write

$$
\begin{aligned}
h\left(z_{1}, z_{2}, z_{3}\right)= & e\left(z_{1}, z_{2}, z_{3} ; R_{1}, R_{2}, R_{3}\right) \\
& -e\left(z_{2}, z_{3} ; R_{2}, R_{3}\right)-e^{\mathrm{at}}\left(z_{1}, R_{1}\right)+h\left(z_{2}, z_{3}\right) .
\end{aligned}
$$

We already discussed $\lim _{z_{2}, z_{3} \rightarrow \infty} h\left(z_{2}, z_{3}\right)$ in the $k=2$ case. The remainder of the right side of (3.11) is, by a similar argument

$$
\int_{0}^{\infty} d s_{1} \psi_{1}\left(s_{1}, \infty, \infty ; R_{1}, R_{2}, R_{3}\right)
$$

where

$$
\begin{aligned}
& \psi_{1}\left(s_{1}, \infty, \infty ; R_{1}, R_{2}, R_{3}\right) \\
& \quad=\lim _{x \rightarrow R_{1}}\left[\phi\left(s_{1}, \infty, \infty ; R_{1}, R_{2}, R_{3} ; x\right)-\phi^{\mathrm{at}}\left(s_{1} ; R_{1} ; x\right)\right]
\end{aligned}
$$

and

$$
\phi\left(s_{1}, \infty, \infty ; R_{1}, R_{2}, R_{3} ; x\right) \equiv \lim _{z_{2}, z_{3} \rightarrow \infty} \phi\left(s_{1}, z_{2}, z_{3} ; R_{1}, R_{2}, R_{3} ; x\right) .
$$

\section{Non-Vanishing of the Three and Higher-Body Interactions}

The $k$-body energy $\varepsilon\left(z_{1}, \ldots, z_{k} ; R_{1}, \ldots, R_{k}\right)$ is defined by successive differences of the total energy $e$. If $a=\left\{a_{1}, \ldots, a_{l}\right\}(l \leqq k)$ is a subset of the integers $K=\{1, \ldots, k\}$, let $e(a)$ denote $e\left(z_{a_{1}}, \ldots, z_{a_{l}}, R_{a_{1}}, \ldots, R_{a_{l}}\right)$, and $|a|=l$ be the cardinality of $a$. Then

$$
\varepsilon(K) \equiv \sum_{\phi \subseteq a \subseteq K}(-1)^{|K|-|a|} e(a)
$$

with $e(\phi) \equiv 0$. Thus,

$$
\varepsilon(\{1,2\})=e\left(z_{1}, z_{2} ; R_{1}, R_{2}\right)-e^{\mathrm{at}}\left(z_{1}\right)-e^{\mathrm{at}}\left(z_{2}\right)=h\left(z_{1}, z_{2} ; R_{1}, R_{2}\right)
$$

is the two-body energy,

$$
\begin{aligned}
\varepsilon(\{1,2,3\})= & e(\{1,2,3\})-e(\{1,2\})-e(\{1,3\}) \\
& -e(\{2,3\})+e^{\text {at }}(\{1\})+e^{\text {at }}(\{2\})+e^{\text {at }}(\{3\})
\end{aligned}
$$


is the three-body energy, and so forth. In terms of $h=e-\sum e^{\text {at }}$, we can also write (4.1), for $|K| \geqq 2$, as

$$
\varepsilon(K)=\sum_{\phi \subseteq a \subseteq K}(-1)^{|K|-|a|} h(a)
$$

since $\sum_{\phi \subseteq b \subseteq K}(-1)^{|b|}=0$ for $|K| \geqq 1$.

It was shown in [2] that the sign of $\varepsilon(K)$ is $(-1)^{|K|}$ for all $\mathbf{z}$ and $\mathbf{R}$. This was done by noting that $\varepsilon(K)=0$ when $z_{1}=0$ and that

$$
\partial \varepsilon(K) / \partial z_{1}=\lim _{x \rightarrow R_{1}} \psi(K ; x)
$$

where

$$
\psi(K ; x)=\sum_{\phi \subseteq a \subseteq K}(-1)^{|K|-|a|} \phi(a ; x) .
$$

It was shown that $(-1)^{|a|} \psi(a ; x) \geqq 0$, for all $x$. Furthermore, it was claimed in [2], but not explicitly proved, that $(-1)^{|a|} \psi(a ; x)>0$, all $x$, including $x=R_{1}$. When $|a|=2$, this is just the strong form of Teller's lemma [1].

As we saw in Sect. III, if $R_{i} \rightarrow l R_{i}$ then $h(a) \rightarrow l^{-7} \Gamma(a)$, with $\Gamma(a)=\Gamma\left(R_{a_{1}}, \ldots, R_{a_{k}}\right)$. Furthermore $\Gamma(a)=\lim _{\boldsymbol{z} \rightarrow \infty} h(a)$. Therefore, the asymptotic form of the $k$-body energy is $l^{-7} \varepsilon_{\infty}(K)$ with

$$
\varepsilon_{\infty}(K)=\sum_{\phi \subseteq a \subseteq K}(-1)^{|K|-|a|} \Gamma(a) .
$$

In [2] we were content to prove that $(-1)^{|K|} \varepsilon(K) \geqq 0$, but now we must prove that $(-1)^{|K|} \varepsilon_{\infty}(K)>0$ in order to be certain that the asymptotic dependence of $\varepsilon(K)$ on $l$ really is $l^{-7}$ and not some higher power of $1 / l$. For $\varepsilon_{\infty}(\{1,2\})=\Gamma(\{1,2\})$, we already know this by the strong form of Teller's lemma. For the same reason, $\Gamma(K)>0$ for all $K$.

In this section we will do two things : (i) We will prove explicitly (by refining the method in [2]) that $\varepsilon_{\infty}(\{1,2,3\})<0$ for all distinct $R_{1}, R_{2}, R_{3}$. The proof can be extended to $|K| \geqq 4$, but it involves the combinatorial lemmas given in [2]. (ii) We will give a simple proof that $\varepsilon_{\infty}(K)$ is not identically zero for all $R_{1}, \ldots, R_{k}$.

To prove that $\varepsilon_{\infty}(\{1,2,3\})<0$, use the expression (3.6) (with $z_{1}=z_{2}=z_{3}=1$ ) in (4.6). Thus, it is sufficient to prove that

$$
\psi(x)=\phi_{123}(x)-\phi_{12}(x)-\phi_{13}(x)+\phi_{1}(x)
$$

has the property that

$$
\lim _{x \rightarrow R_{1}} \psi(x)<0 .
$$

In (4.7) the notation is that $\phi_{123}(x)=\phi\left(\lambda, \lambda, \lambda ; R_{1}, R_{2}, R_{3} ; x\right)$, etc. If (4.8) is true for all $\lambda>0$ then integration with respect to $\lambda$ gives $\varepsilon_{\infty}(\{1,2,3\})<0$. From now on we set $\lambda=1$ for simplicity. 
By the TF equation (1.4), $\psi$ is continuous everywhere since the $\left|x-R_{i}\right|^{-1}$ singularities cancel (see [1]). We show that $\psi(x)<0$ everywhere as follows:

$$
-(4 \pi)^{-1} \Delta \psi=-\phi_{123}^{3 / 2}+\phi_{12}^{3 / 2}+\phi_{13}^{3 / 2}-\phi_{1}^{3 / 2}
$$

everywhere in $\mathbb{R}^{3}$.

First observe that if $a, b, c, d$ are real positive numbers such that

$$
d \leqq(b \text { and } c) \leqq a
$$

then

$$
b^{3 / 2}+c^{3 / 2} \leqq a^{3 / 2}+d^{3 / 2}+\frac{3}{2} a^{1 / 2}(b+c-a-d),
$$

where

$$
(x)^{+}=(|x|+x) / 2 .
$$

This is an easy consequence of the convexity of the function $t \rightarrow t^{3 / 2}$. If $a=\phi_{123}$, $b=\phi_{12}, c=\phi_{13}$ and $d=\phi_{1}$, the inequality (4.10) (for each $x \in \mathbb{R}^{3}$ ) is just Teller's lemma. Therefore we find,

$$
-(4 \pi)^{-1} \Delta \psi \leqq(3 / 2) \phi_{123}^{1 / 2}(-\psi)^{+},
$$

that is

$$
-(4 \pi)^{-1} \Delta \psi+c(x) \psi \leqq 0
$$

where $c(x)=(3 / 2) \phi_{123}^{1 / 2}(x)$ on the set $\{x \mid \psi(x)<0\}$ and $c(x)=0$ on the set $\{x \mid \psi(x) \geqq 0\}$. Since [1] each $\phi$ behaves like $\left|x-R_{i}\right|^{-1}$ near the singularities, $\Delta \psi \in L_{\mathrm{loc}}^{p}$ for every $p<2$. It follows from the $L^{p}$ regularity theorem that $\psi \in H_{\mathrm{loc}}^{1}$; also $c(x) \in L_{\mathrm{loc}}^{p}$ for every $p<6$ and in particular for some $p>3 / 2$. In addition $\psi(x) \rightarrow 0$ as $|x| \rightarrow \infty$; the strong maximum principle (see [7]) implies therefore that either $\psi<0$ everywhere or $\psi \equiv 0$. If $\psi \equiv 0$ we would have $\phi_{12}+\phi_{13} \equiv \phi_{123}+\phi_{1}$ and $\phi_{12}^{3 / 2}+\phi_{13}^{3 / 2} \equiv \phi_{123}^{3 / 2}+\phi_{1}^{3 / 2}$. This is possible only if $\phi_{123}=\phi_{12}$ or if $\phi_{123}=\phi_{13}$. Since $\phi_{123}>\phi_{12}$ and $\phi_{123}>\phi_{13}$ for all $x$ (strong form of Teller's lemma: [1], Theorem V.6) we conclude that $\psi(x)<0$ for all $x$.

As remarked before, a similar argument, together with the combinatorial lemmas of [2], proves that $(-1)^{|K|} \psi(K ; x)$ (which is a continuous function) is strictly positive. Thus $(-1)^{|K|} \varepsilon_{\infty}(K)>0$.

We turn now to the alternative proof that $\varepsilon_{\infty}(K)$ is not identically zero. It hinges on the following:

Lemma 1. Let $R_{1}, \ldots, R_{k-1}$ be given and suppose that $R_{k}^{(n)}$ is a sequence of vectors such that $R_{k}^{(n)} \rightarrow R_{k-1}$. Then

$$
\Gamma\left(R_{1}, \ldots, R_{k-1}, R_{k}^{(n)}\right)-\Gamma\left(R_{k-1}, R_{k}^{(n)}\right) \rightarrow \Gamma\left(R_{1}, \ldots, R_{k-1}\right),
$$

and

$$
\Gamma\left(R_{1}, \ldots, R_{k-2}, R_{k}^{(n)}\right) \rightarrow \Gamma\left(R_{1}, \ldots, R_{k-1}\right) .
$$

To use Lemma 1 , suppose $|K| \geqq 3$ and let $\varepsilon_{\infty}^{(n)} \equiv \varepsilon_{\infty}\left(R_{1}, \ldots, R_{k-1}, R_{k}^{(n)}\right)$. Then, since $\sum_{\{k-1, k\} \subseteq a \subseteq K}(-1)^{|a|}=0$, we have from (4.6) and Lemma 1: 
Lemma 2. If $R_{k}^{(n)} \rightarrow R_{k-1}$, and if $k \geqq 3$, then $\varepsilon_{\infty}\left(R_{1}, \ldots, R_{k-1}, R_{k}^{(n)}\right) \rightarrow-\varepsilon_{\infty}\left(R_{1}, \ldots, R_{k-1}\right)$.

Corollary. Since $\varepsilon_{\infty}(\{1,2\})=\Gamma(\{1,2\})>0, \varepsilon_{\infty}(K)$ is not identically zero.

The proof of (4.12) is easy. One can use (3.6), together with the continuity of the TF $\phi$ in $R_{k}$ and dominated convergence. Alternatively, one can use (3.10)-(3.14) and the method of Proposition A.6 in the appendix. Next we consider (4.11).

(4.11) is empty if $|K| \leqq 2$. If $|K| \geqq 3$, write the left side of (4.11) as $\sum_{j=1}^{k-2} \Delta_{j}(n)$ where $\Delta_{j}(n)=\Gamma\left(R_{j}, \ldots, R_{k-1}, R_{k}^{(n)}\right)-\Gamma\left(R_{j+1}, \ldots, R_{k-1}, R_{k}^{(n)}\right)$.

We claim $\Delta_{j}(n) \rightarrow \Gamma\left(R_{j}, \ldots, R_{k-1}\right)-\Gamma\left(R_{j+1}, \ldots, R_{k-1}\right) \equiv \Delta_{j}$, for $j<k-2$, while $\Delta_{k-2}(n) \rightarrow \Gamma\left(R_{k-2}, R_{k-1}\right) \equiv \Delta_{k-2}$ in which case we are done. Now, as shown at the end of Sect. III,

$$
\begin{aligned}
\Delta_{j}(n) & =\int_{0}^{\infty} d z_{j} \lim _{x \rightarrow R_{J}} \psi_{j}\left(z_{j} ; x ; n\right) \\
\psi_{j}\left(z_{j} ; x ; n\right) & =\phi\left(z_{j}, \infty, \ldots, \infty ; R_{j}, \ldots, R_{k}^{(n)} ; x\right)-\phi^{\mathrm{at}}\left(z_{j} ; R_{j} ; x\right)
\end{aligned}
$$

while

$$
\begin{aligned}
\Delta_{j} & =\int_{0}^{\infty} d z_{j} \lim _{x \rightarrow R_{j}} \psi_{j}\left(z_{j} ; x\right) \\
\psi_{j}\left(z_{j} ; x\right) & =\phi\left(z_{j}, \infty, \ldots, \infty ; R_{j}, \ldots, R_{k-1} ; x\right)-\phi^{\mathrm{at}}\left(z_{j} ; R_{j} ; x\right) .
\end{aligned}
$$

To complete the proof of (4.11) we use Proposition A.6 together with dominated convergence.

\section{Appendix}

Our main purpose here is to study the function $\phi\left(z_{1}, z_{2}, \ldots, z_{k} ; R_{1}, \ldots, R_{k} ; x\right)$ as some of the $z_{i} \rightarrow \infty$. This will lead to a TF differential equation with "strong singularities" and we will study existence, uniqueness and other properties of these equations. Some of these results were alluded to in the main text.

We begin with a Lemma about solutions to the TF equation away from singularities (results of this kind, which are due to H.B., are quoted in [8]).

Lemma A.1. Let $B_{R}$ be the open ball of radius $R$ in $\mathbb{R}^{3}$ and suppose $\phi(x) \geqq 0$ is locally bounded in $B_{R}$ and satisfies the TF differential equation

$$
-(4 \pi)^{-1} \Delta \phi+\phi^{3 / 2}=0
$$

in $B_{R}$. Then $\phi(0) \leqq c R^{-4}$ for some universal constant c. In particular $c \leqq(20 / \pi)^{2}$. (We do not know the best constant c.)

Proof. Consider the function $\psi(x)=c R^{4}\left[R^{2}-|x|^{2}\right]^{-4}$ in $B_{R}$. It is easy to compute that $-(4 \pi)^{-1} \Delta \psi+\psi^{3 / 2} \geqq 0$ in $B_{R}$ provided $c \geqq(20 / \pi)^{2}$. We claim that $\phi \leqq \psi$. First, 
we can always assume that $\phi$ is bounded on $B_{R}$ (replace $R$ by $R-\varepsilon$ ). Then $-(4 \pi)^{-1} \Delta(\phi-\psi)+\phi^{3 / 2}-\psi^{3 / 2} \leqq 0$ and therefore

$$
-\frac{1}{4 \pi} \int \Delta(\phi-\psi)(\phi-\psi)^{+}+\int\left(\phi^{3 / 2}-\psi^{3 / 2}\right)(\phi-\psi)^{+} \leqq 0 .
$$

Since $(\phi-\psi)^{+}=0$ on the boundary of $B_{R}$ we have

$$
-\frac{1}{4 \pi} \int \Delta(\phi-\psi)(\phi-\psi)^{+}=\frac{1}{4 \pi} \int_{[\phi \geqq \psi]}|\nabla(\phi-\psi)|^{2} \geqq 0 .
$$

Therefore

$$
\int\left(\phi^{3 / 2}-\psi^{3 / 2}\right)(\phi-\psi)^{+}=0
$$

and thus $\phi \leqq \psi$ a.e. However $\Delta \phi$ is bounded and so $\phi$ is continuous. Hence $\phi(0) \leqq \psi(0)=c R^{-4}$.

Corollary A.2. Suppose $\phi$ satisfies the TF differential Eq. (A.1) outside some bounded set. Then $\phi(x)$ must go to zero at infinity at least as fast as $c|x|^{-4}$.

Next, we consider the behavior of $\phi\left(z_{1}, \ldots, z_{k} ; R_{1}, \ldots, R_{k} ; x\right)$ as $z_{2}, \ldots, z_{k} \rightarrow \infty$ for fixed $z_{1}, R_{1}, R_{2}, \ldots, R_{k}$ (we assume, of course, that the $R_{i}$ are distinct). Our main result is the following

Proposition A.3. As $z_{2}, \ldots, z_{k} \uparrow+\infty, \phi\left(z_{1}, \ldots, z_{k} ; R_{1}, \ldots, R_{k} ; x\right) \uparrow \phi(x)$ where $\phi(x)$ is the unique positive function which is locally bounded except at the $R_{i}$ and satisfies, in the sense of distributions, the TF equation:

$$
-(4 \pi)^{-1} \Delta \phi+\phi^{3 / 2}=0
$$

in $\mathbb{R}^{3} \backslash \bigcup_{i=1}^{k}\left\{R_{i}\right\}$, and

$$
\left.\begin{array}{l}
\left|\phi(x)-z_{1}\right| x-\left.R_{1}\right|^{-1} \mid \text { remains bounded as } x \rightarrow R_{1}, \\
\left|\phi(x)-(3 / \pi)^{2}\right| x-\left.R_{j}\right|^{-4} \mid \text { remains bounded as } x \rightarrow R_{j}(j>1) .
\end{array}\right\}
$$

In fact

$$
0 \leqq \phi(x)-(3 / \pi)^{2}\left|x-R_{j}\right|^{-4} \leqq c\left|x-R_{j}\right|^{3}
$$

as $x \rightarrow R_{j}(j>1)$ for some constant $c$ (which can depend only on $\min _{i \neq j}\left|R_{i}-R_{j}\right|$ ).

Proof. We will give the proof for $k=2$; the extension is trivial. We know (see [1]) that

$$
\begin{aligned}
& \phi\left(z_{2} ; R_{2} ; x\right) \leqq \phi\left(z_{1}, z_{2} ; R_{1}, R_{2} ; x\right) \leqq \phi\left(z_{1} ; R_{1} ; x\right)+\phi\left(z_{2} ; R_{2} ; x\right) \\
& \phi\left(z_{1} ; R_{1} ; x\right) \leqq \phi\left(z_{1}, z_{2} ; R_{1}, R_{2} ; x\right) \leqq \phi\left(z_{1} ; R_{1} ; x\right)+\phi\left(z_{2} ; R_{2} ; x\right) .
\end{aligned}
$$

By scaling and by Sommerfeld's asymptotic formula [5] (see [1])

$$
\phi\left(z_{2} ; R_{2} ; x\right) \uparrow(3 / \pi)^{2}\left|x-R_{2}\right|^{-4} \text { as } z_{2} \rightarrow \infty,
$$


if $x \neq R_{2}$. Consequently (A.2) and (A.3) follow immediately (use monotone or dominated convergence theorem). We show now that the solution of (A.2) and (A.3) is unique. Assume $\phi \geqq 0, \bar{\phi} \geqq 0$ are two solutions of (A.2), (A.3) which are bounded except near $R_{1}$ and $R_{2}$. It follows from standard elliptic regularity theory that $\phi, \bar{\phi}$ are $C^{2}$ except near $R_{1}$ and $R_{2}$. Let $\xi \geqq 0$ be a smooth function with compact support such that $R_{1} \notin \operatorname{supp}(\xi), R_{2} \notin \operatorname{supp}(\xi)$. We have upon multiplication of the difference of the corresponding equations (A.2) by $\xi(\phi-\bar{\phi})$, and integration by parts, that

$$
\begin{aligned}
& (4 \pi)^{-1} \int \sum_{i}\left|\frac{\partial}{\partial x_{i}}(\phi-\bar{\phi})\right|^{2} \xi+(4 \pi)^{-1} \int \sum_{i}\left[\frac{\partial}{\partial x_{i}}(\phi-\bar{\phi})\right](\phi-\bar{\phi}) \frac{\partial \xi}{\partial x_{i}} \\
& \quad+\int\left(\phi^{3 / 2}-\bar{\phi}^{3 / 2}\right)(\phi-\bar{\phi}) \xi=0 .
\end{aligned}
$$

Therefore, integrating by parts again,

$$
\int\left(\phi^{3 / 2}-\bar{\phi}^{3 / 2}\right)(\phi-\bar{\phi}) \xi \leqq \frac{1}{8 \pi} \int|\phi-\bar{\phi}|^{2} \Delta \xi .
$$

We now choose $\xi=\xi_{n}$ where $\xi_{n} \uparrow 1$ and $\xi_{n}$ has the following properties:

$$
\begin{aligned}
& 0 \leqq \xi_{n} \leqq 1, \\
& \xi_{n}(x)=0 \quad \text { outside } B_{n+1}(0) \text { and on } B_{1 / 2 n}\left(R_{1}\right) \cup B_{1 / 2 n}\left(R_{2}\right), \\
& \xi_{n}(x)=1 \quad \text { on } B_{n}(0) \backslash\left[B_{1 / n}\left(R_{1}\right) \cup B_{1 / n}\left(R_{2}\right)\right],
\end{aligned}
$$

where $B_{r}(R)=\{x|| x-R \mid<r\}$, and

$$
\begin{aligned}
& \left|\Delta \xi_{n}\right| \leqq C n^{2} \quad \text { on } \quad B_{1 / n}\left(R_{1}\right) \cup B_{1 / n}\left(R_{2}\right), \\
& \left|\Delta \xi_{n}\right| \leqq C \text { for } \quad n<|x|<n+1,
\end{aligned}
$$

with $C$ independent of $n$.

Hence we find

$$
\begin{aligned}
& 8 \pi \int\left(\phi^{3 / 2}-\bar{\phi}^{3 / 2}\right)(\phi-\bar{\phi}) \xi_{n} \leqq C n^{2} \int_{B_{1 / n}\left(R_{1}\right)}|\phi-\bar{\phi}|^{2} \\
& +C n^{2} \int_{B_{1 / n}\left(R_{2}\right)}|\phi-\bar{\phi}|^{2}+C \int_{n<|x|<n+1}|\phi-\bar{\phi}|^{2} .
\end{aligned}
$$

As $n \rightarrow \infty$ the right side goes to zero (since $|\phi-\bar{\phi}|$ is bounded near $R_{1}$ and $R_{2}$, and $|\phi-\bar{\phi}|$ goes to zero like $|x|^{-4}$ at infinity (see Corollary A.2). Thus $\phi=\bar{\phi}$ a.e. The fact that

$$
0 \leqq \phi(x)-(3 / \pi)^{2}\left|x-R_{2}\right|^{-4}
$$

follows by taking the limit in (A.5). The other inequality in (A.4) is a consequence of our next proposition.

Proposition A.4. Assume $\phi \geqq 0, \phi$ is locally bounded on the set $\{x|0<| x \mid<2 R\}$ and $\phi$ satisfies

$$
-(4 \pi)^{-1} \Delta \phi+\phi^{3 / 2}=0
$$


in the sense of distributions on $\{x|0<| x \mid<2 R\}$. Then

$$
\phi(x) \leqq(3 / \pi)^{2}|x|^{-4}+\frac{c|x|^{3}}{R^{7}} \text { for }|x|<R,
$$

where $c$ is the same universal constant as in Lemma A.1.

Proof. By scaling we can always assume that $R=1$. The proof is divided into three steps.

Step 1. Set $\psi(x)=(3 / \pi)^{2}|x|^{-4}$ and $u=\max \{\phi, \psi\}$. Then we have

$$
-(4 \pi)^{-1} \Delta u+u^{3 / 2} \leqq 0 \quad \text { on }\{x|0<| x \mid<2\} .
$$

This is a consequence of the following:

$$
-(4 \pi)^{-1} \Delta \psi+\psi^{3 / 2}=0 \text { for }|x|>0 .
$$

Since $u=(1 / 2)(\phi+\psi+|\phi-\psi|)$, we deduce from Kato's inequality (see [12]) that

$$
\begin{aligned}
\Delta u & \geqq(1 / 2)(\Delta \phi+\Delta \psi+\operatorname{sign}(\phi-\psi) \Delta(\phi-\psi)) \\
& =(4 \pi / 2)\left(\phi^{3 / 2}+\psi^{3 / 2}+\left|\phi^{3 / 2}-\psi^{3 / 2}\right|\right) \\
& =4 \pi \max \left\{\phi^{3 / 2}, \psi^{3 / 2}\right\}=4 \pi u^{3 / 2} .
\end{aligned}
$$

Step 2. Let $\bar{u}(r)=\frac{1}{4 \pi r^{2}} \int_{|x|=r} u$ be the spherical average of $u$. We shall prove that

$$
\bar{u}(r) \leqq(3 / \pi)^{2} r^{-4}+c r^{3}, \text { for } 0<r<1,
$$

with the universal constant $c \leqq(20 / \pi)^{2}$. Indeed by averaging (A.7) we find

$$
+(4 \pi)^{-1}\left(\bar{u}^{\prime \prime}+\frac{2}{r} \bar{u}^{\prime}\right)+\overline{u^{3 / 2}} \leqq 0, \text { for } 0<r<2 .
$$

By the convexity of the function $t \mapsto t^{3 / 2}$ we have $\bar{u}^{3 / 2} \leqq \overline{u^{3 / 2}}$ and thus for $0<r<2$

$$
-(4 \pi)^{-1}\left(\bar{u}^{\prime \prime}+\frac{2}{r} \bar{u}^{\prime}\right)+\bar{u}^{3 / 2} \leqq 0 .
$$

We now introduce the function $v(r)$ defined by the relation

$$
v\left(r^{7}\right)=(\pi / 3)^{2} r^{4} \bar{u}(r), \quad 0<r<2 .
$$

It follows from the definition of $u$ that $v \geqq 1$ and by Lemma A.1, $v\left(r^{7}\right) \leqq c(\pi / 3)^{2}$ for $0<r<1$. Using the relation

$$
\bar{u}(r)=(3 / \pi)^{2} r^{-4} v\left(r^{7}\right)
$$

and (A.9) we obtain

$$
-r^{14} v^{\prime \prime}\left(r^{7}\right)+(12 / 49) v\left(r^{7}\right)\left(\left|v\left(r^{7}\right)\right|^{1 / 2}-1\right) \leqq 0 .
$$

With $t=r^{7}$ we find for $0<t<2^{7}$

$$
-t^{2} v^{\prime \prime}+(12 / 49) v\left(v^{1 / 2}-1\right) \leqq 0 \text {. }
$$


It follows in particular that $v$ is convex. By Lemma A.1 $v(t)$ remains bounded as $t \rightarrow 0$. Thus $\lim _{t \rightarrow 0} v(t)=v(0)$ exists. We must have $v(0)=1$; otherwise for small $t$ (A.11) leads to $v^{\prime \prime}(t) \geqq \alpha t^{-2}$ with some $\alpha>0$ and thus $v(t) \geqq c-\alpha \log t$, which contradicts the boundedness of $v$. We deduce from the convexity of $v$ that $v(t) \leqq 1+c(\pi / 3)^{2} t$, for $0<t<1$, since $v(1) \leqq c(\pi / 3)^{2}$. By (A.10) we have now for $0<r<1$

$$
\bar{u}(r) \leqq(3 / \pi)^{2} r^{-4}+c r^{3} .
$$

Step 3. For $|x|<1$ define

$$
(3 / \pi)^{2}|x|^{-4}+c|x|^{3} \equiv \omega(x) \text {. }
$$

It suffices to prove that $u=\max \{\phi, \psi\} \leqq \omega$. For $|x|=1 \quad u(x) \leqq \omega(x)$ because $\psi(x) \leqq \omega(x)$ and $\phi(x) \leqq \omega(x)$ by Lemma A.1. Next we observe that

$$
-(4 \pi)^{-1} \Delta \omega+\omega^{3 / 2} \geqq 0 \text { for }|x|>0 .
$$

Indeed we have

$$
\begin{aligned}
-(4 \pi)^{-1} \Delta \omega+\omega^{3 / 2}= & -(3 / \pi)\left[(3 / \pi)^{2} r^{-6}+c r\right] \\
& +\left((3 / \pi)^{2} r^{-4}+c r^{3}\right)^{3 / 2} \geqq(3 / 2 \pi) c r \geqq 0,
\end{aligned}
$$

[using the fact that $\left.(1+a)^{3 / 2} \geqq 1+(3 / 2) a\right]$. Combining (A.7) and (A.13) we find for $0<|x|<2$

$$
-(4 \pi)^{-1} \Delta(u-\omega)+u^{3 / 2}-\omega^{3 / 2} \leqq 0 .
$$

Let $\xi_{n}(x)$ be a sequence of smooth functions such that $0 \leqq \xi_{n} \leqq 1$,

$$
\begin{aligned}
& \xi_{n}(x)=\left\{\begin{array}{lll}
1 & \text { for } & |x|>\frac{1}{n} \\
0 & \text { for } & |x|<\frac{1}{2 n}
\end{array}\right. \\
& \left|\Delta \xi_{n}\right| \leqq C n^{2} .
\end{aligned}
$$

Let $\theta(t)$ be a smooth function such that $\theta^{\prime} \geqq 0, \theta(t)=0$ for $t \leqq 0, \theta(t)>0$ for $t>0$, $\theta(t) \leqq 1$ for $t \geqq 1$. Set $j(t)=\int_{0}^{t} \theta(s) d s$ so that $0 \leqq j(t) \leqq t^{+}$for all $t$. Multiplying (A.14) by $\xi_{n}(x) \theta(u-\omega)$ and integrating by parts on $|x|<1$ we find (using the fact that $u-\omega \leqq 0$ on $|x|=1)$

$$
\begin{aligned}
& (4 \pi)^{-1} \int_{|x|<1} \nabla(u-\omega) \cdot \nabla \xi_{n} \theta(u-\omega)+(4 \pi)^{-1} \int_{|x|<1}|\nabla(u-\omega)|^{2} \xi_{n} \theta^{\prime}(u-\omega) \\
& \quad+\int_{|x|<1}\left(u^{3 / 2}-\omega^{3 / 2}\right) \xi_{n} \theta(u-\omega) \leqq 0 .
\end{aligned}
$$

Using the relation $\nabla(u-\omega) \theta(u-\omega)=\nabla j(u-\omega)$ and integrating by parts again we obtain

$$
\int_{|x|<1}\left(u^{3 / 2}-\omega^{3 / 2}\right) \xi_{n} \theta(u-\omega) \leqq(4 \pi)^{-1} \int_{|x|<1} j(u-\omega) \Delta \xi_{n} \leqq C n^{2} \int_{\frac{1}{2 n}<|x|<\frac{1}{n}} j(u-\omega) .
$$


But $j(u-\omega) \leqq(u-\omega)^{+} \leqq(u-\psi)^{+}=u-\psi($ since $\psi \leqq \omega)$. Consequently,

$$
\begin{gathered}
\int_{|x|<1}\left(u^{3 / 2}-\omega^{3 / 2}\right) \xi_{n} \theta(u-\omega) \leqq C n^{2} \int_{\frac{1}{2 n}<|x|<\frac{1}{n}}(u-\psi) \\
=C n^{2} \int_{\frac{1}{2 n}}^{\frac{1}{n}}(\bar{u}-\psi) 4 \pi r^{2} d r \leqq C n^{2} \int_{\frac{1}{2 n}}^{\frac{1}{n}} r^{5} d r
\end{gathered}
$$

by Step 2. As $n \rightarrow \infty$ we obtain

$$
\int_{|x|<1}\left(u^{3 / 2}-\omega^{3 / 2}\right) \theta(u-\omega) \leqq 0
$$

and therefore $u \leqq \omega$ for $|x|<1$.

Remark. One can improve the conclusion of Proposition A.4. In fact, let $p$ denote the positive solution of $p(p+1)=18(p>3)$. Assume (A.6) holds with $R=1$. Then

$$
\phi(x) \leqq\left(\frac{3}{\pi}\right)^{2}|x|^{-4}+c|x|^{p} \equiv \tilde{\omega}(x)
$$

for $|x|<1$. Indeed, using the same argument as in Step 3, it suffices to verify that $\tilde{\omega}(x)$ satisfies

$$
-(4 \pi)^{-1} \Delta \tilde{\omega}+\tilde{\omega}^{3 / 2} \geqq 0
$$

which follows from an easy computation.

Next we prove Proposition A.5 about the interchange of limits that was used at the end of Sect. III and which will also be used in Proposition A.6.

\section{Proposition A.5.}

$$
\begin{array}{r}
\lim _{z_{2} \rightarrow \infty} \lim _{x \rightarrow R_{1}}\left[\phi\left(z_{1}, z_{2}, \ldots, z_{k} ; R_{1}, R_{2}, \ldots, R_{k} ; x\right)-\phi^{\mathrm{at}}\left(z_{1} ; R_{1} ; x\right)\right] \\
\quad=\lim _{x \rightarrow R_{1}}\left[\phi\left(z_{1}, \infty, \ldots, \infty ; R_{1}, R_{2}, \ldots, R_{k} ; x\right)-\phi^{\mathrm{at}}\left(z_{1} ; R_{1} ; x\right)\right],
\end{array}
$$

Proof. For simplicity we assume $k=2$.

Step 1 . The right side of the last equation exists. To simplify matters set:

$$
\begin{aligned}
\phi(x) & =\phi\left(z_{1}, \infty ; R_{1}, R_{2} ; x\right) \\
\phi^{(n)}(x) & =\phi\left(z_{1}, n ; R_{1}, R_{2} ; x\right) \\
\phi^{\mathrm{at}}(x) & =\phi^{\mathrm{at}}\left(z_{1} ; R_{1} ; x\right) .
\end{aligned}
$$

We know that in the sense of distributions in $\mathbb{R}^{3} \backslash\left\{R_{2}\right\}$

$$
-\frac{1}{4 \pi} \Delta \phi^{(n)}+\left[\phi^{(n)}\right]^{3 / 2}=z_{1} \delta\left(x-R_{1}\right) .
$$

Recall that [1]

$$
0 \leqq \phi^{(n)}(x)-\phi^{\mathrm{at}}(x) \leqq\left(\frac{3}{\pi}\right)^{2}\left|x-R_{2}\right|^{-4} .
$$


Clearly

$$
\phi^{\mathrm{at}}(x)+\left(\frac{3}{\pi}\right)^{2}\left|x-R_{2}\right|^{-4} \in L_{\mathrm{loc}}^{3 / 2}\left(\mathbb{R}^{3} \backslash\left\{R_{2}\right\}\right) .
$$

Therefore we can pass to the limit in (A.15) using dominated convergence. This leads to

$$
-\frac{1}{4 \pi} \Delta \phi+\phi^{3 / 2}=z_{1} \delta\left(x-R_{1}\right)
$$

in the sense of distributions in $\mathbb{R}^{3} \backslash\left\{R_{2}\right\}$. It follows that

$$
-\frac{1}{4 \pi} \Delta\left(\phi-\phi^{\mathrm{at}}\right)+\phi^{3 / 2}-\left(\phi^{\mathrm{at}}\right)^{3 / 2}=0
$$

in the sense of distributions in $\mathbb{R}^{3} \backslash\left\{R_{2}\right\}$.

Finally observe that $\phi^{3 / 2}-\left(\phi^{\text {at }}\right)^{3 / 2} \in L_{\text {loc }}^{p}\left(\mathbb{R}^{3} \backslash\left\{R_{2}\right\}\right)$ for each $p<2$. Now we rely on the regularity theory for elliptic equations: if a function $u$ satisfies $\Delta u \in L_{\text {loc }}^{p}(\Omega)$ with $p>\frac{n}{2}$ on an open set $\Omega \subset \mathbb{R}^{n}$ then $u$ is continuous on $\Omega$.

It follows that $\phi-\phi^{\text {at }}$ is continuous on $\mathbb{R}^{3} \backslash\left\{R_{2}\right\}$.

Step 2. Recall Dini's Lemma: Let $f_{n}(x)$ be a sequence of continuous functions on a compact space $K$ such that $f_{n}(x) \uparrow f(x)$ (pointwise) as $n \rightarrow \infty$. If $f(x)$ is continuous, then in fact $f_{n} \rightarrow f$ uniformly in $K$.

[In particular $\lim _{x \rightarrow \xi} \lim _{n \rightarrow \infty} f_{n}(x)=\lim _{n \rightarrow \infty} \lim _{x \rightarrow \xi} f_{n}(x)$ ].

In our context choose

$$
\begin{aligned}
& f_{n}(x)=\phi^{(n)}(x)-\phi^{\mathrm{at}}(x) \\
& f(x)=\phi(x)-\phi^{\mathrm{at}}(x)
\end{aligned}
$$

and $K$ any small ball around $R_{1}$.

Finally, we consider the behavior of the solution of the TF Eq. (A.2), (A.3) with strong singularities as two of the strong singularities coalesce.

Proposition A.6. Suppose $k \geqq 3$ and $R_{1}, \ldots, R_{k-1}, z_{1}$ are given. Let $R_{k}^{(n)}$ be a sequence of points in $\mathbb{R}^{3}$ such that $R_{k}^{(n)} \rightarrow R_{k-1}$ as $n \rightarrow \infty$. If $\phi_{n}(x)$ is the solution to (A.2), (A.3) for $R_{1}, \ldots, R_{k}^{(n)}$ and $\phi(x)$ is the solution for $R_{1}, \ldots, R_{k-1}$ then $\phi_{n}(x) \rightarrow \phi(x)$ as $n \rightarrow \infty$ $\left(x \neq R_{i}\right)$. Moreover,

$$
\lim _{x \rightarrow R_{1}}\left[\phi_{n}(x)-\phi^{\mathrm{at}}\left(z_{1} ; R_{1} ; x\right)\right] \rightarrow \lim _{x \rightarrow R_{1}}\left[\phi(x)-\phi^{\mathrm{at}}\left(z_{1} ; R_{1} ; x\right)\right] .
$$

Proof. For simplicity, we give the proof for $k=3$. As in the proof of Proposition A.3 we have

$$
(3 / \pi)^{2}\left|x-R_{2}\right|^{-4} \leqq \phi_{n}(x), \quad \forall x .
$$

Also we have [1]

$$
\phi^{\mathrm{at}}\left(z_{1} ; R_{1} ; x\right) \leqq \phi_{n}(x) \leqq \phi^{\mathrm{at}}\left(z_{1} ; R_{1} ; x\right)+(3 / \pi)^{2}\left[\left|x-R_{2}\right|^{-4}+\left|x-R_{3}^{(n)}\right|^{-4}\right] .
$$

It follows that $\phi_{n}$ is bounded on every compact subset $K$ of $\mathbb{R}^{3} \backslash\left\{R_{1}\right\} \cup\left\{R_{2}\right\}$ and so is $\Delta \phi_{n}$. Therefore $\phi_{n}$ is bounded in $C^{1}(K)$ and by the Theorem of Ascoli we may 
extract a subsequence $\phi_{n_{k}}$ which converges to a limit $\phi$ uniformly on every compact subset of $\mathbb{R}^{3} \backslash\left\{R_{1}\right\} \cup\left\{R_{2}\right\}$. Clearly $\phi$ satisfies

$$
-(4 \pi)^{-1} \Delta \phi+\phi^{3 / 2}=0
$$

in the sense of distributions on $\mathbb{R}^{3} \backslash\left\{R_{1}\right\} \cup\left\{R_{2}\right\}$. In addition we deduce from (A.17) that $\left|\phi(x)-z_{1}\right| x-\left.R_{1}\right|^{-1} \mid$ remains bounded as $x \rightarrow R_{1}$. Also, we know by Proposition A.4 that

$$
\phi(x) \leqq(3 / \pi)^{2}\left|x-R_{2}\right|^{-4}+c
$$

for $x$ near $R_{2}$. Combining (A.16) and (A.18) we see that

$$
\left|\phi(x)-(3 / \pi)^{2}\right| x-\left.R_{2}\right|^{-4} \mid
$$

remains bounded as $x \rightarrow R_{2}$. We know by Proposition A.3 that these conditions determine $\phi$ uniquely and thus $\phi$ must coincide with the solution obtained in Proposition A.3.

Acknowledgements. One of the authors (E.L.) would like to thank the following for their hospitality during the course of this work: The Institut des Hautes Etudes Scientifiques, Bures-sur-Yvette and the College de France. We thank Rafael Benguria for valuable help and for drawing our attention to Ref. [10].

\section{References}

1. Lieb, E.H., Simon, B. : The Thomas-Fermi theory of atoms, molecules and solids. Adv. Math. 23, 22-116 (1977). See also Brezis, H., Benilan, Ph. : Non-linear problems related to the Thomas-Fermi equation (in preparation); Lieb, E.H., Simon, B. : Thomas-Fermi theory revisited. Phys. Rev. Lett. 31, 681-683 (1973); Lieb, E.H.: Thomas-Fermi and Hartree-Fock theory. Proc. Int. Congr. of Math., Vancouver (1974); Lieb, E.H.: The stability of matter. Rev. Mod. Phys. 48, 553-569 (1976)

2. Benguria, R., Lieb, E.H.: Many-body atomic potentials in Thomas-Fermi theory. Ann. Phys. NY 110, 34-45 (1978)

3. Benguria, R., Lieb, E.H.: The positivity of the pressure in Thomas-Fermi theory. Commun. math. Phys. 63, 193-218 (1978)

4. Lee, C.E., Longmire, C.L., Rosenbluth, M.N.: Thomas-Fermi calculation of potential between atoms. Los Alamos Scientific Laboratory report (LA-5694-MS) (1974)

5. Sommerfeld, A.: Asymptotische Integration der Differentialgleichung des Thomas-Fermischen Atoms. Z. Physik 78, 283-308 (1932)

6. Teller, E.: On the stability of molecules in the Thomas-Fermi theory. Rev. Mod. Phys. 34, 627-631 (1962)

7. Trudinger, N.: Linear elliptic operators with measurable coefficients. Ann. Sc. Norm. Sup. Pisa, Ser. 3, 27, 265-308 (1973)

8. Nirenberg, L.: Non-linear differential equations invariant under certain geometric transformations. Istit. Naz. di Alta Mat. (Bologna), Symp. Math. 18, 399-405 (1976)

9. Casimir, H.B.C., Polder, D.: The influence of retardation on the London-van der Waals forces. Phys. Rev. 73, 360-372 (1948)

10. Roberts, R.E.: Upper- and lower-bound energy calculations for atoms and molecules in the Thomas-Fermi theory. Phys. Rev. 170, 8-11 (1968)

11. Firsov, O.B.: Interaction energy of atoms for small nuclear separations. Zh. Eksp. Teor. Fiz. 32, 1464 (1957). English translation: Soviet Phys. JETP 5, 1192-1196 (1957)

12. Kato, T.: Schrödinger operators with singular potentials. Isr. J. Math. 13, 135-148 (1973)

Communicated by J. Glimm

Received September 19, 1978 